\title{
A genomic and evolutionary approach reveals non-genetic drug resistance in malaria
}

\author{
Jonathan D Herman 1,2,3,4,5, Daniel P Rice ${ }^{6,7}$, Ulf Ribacke ${ }^{1,8}$, Jacob Silterra², Amy A Deik², Eli L Moss², \\ Kate M Broadbent ${ }^{2,6}$, Daniel E Neafsey ${ }^{1,2}$, Michael M Desai ${ }^{6,7}$, Clary B Clish ${ }^{2}$, Ralph Mazitschek ${ }^{1,2,9}$ \\ and Dyann F Wirth ${ }^{1,2^{*}}$
}

\begin{abstract}
Background: Drug resistance remains a major public health challenge for malaria treatment and eradication. Individual loci associated with drug resistance to many antimalarials have been identified, but their epistasis with other resistance mechanisms has not yet been elucidated.

Results: We previously described two mutations in the cytoplasmic prolyl-tRNA synthetase (cPRS) gene that confer resistance to halofuginone. We describe here the evolutionary trajectory of halofuginone resistance of two independent drug resistance selections in Plasmodium falciparum. Using this novel methodology, we discover an unexpected non-genetic drug resistance mechanism that $P$. falciparum utilizes before genetic modification of the CPRS. $P$. falciparum first upregulates its proline amino acid homeostasis in response to halofuginone pressure. We show that this non-genetic adaptation to halofuginone is not likely mediated by differential RNA expression and precedes mutation or amplification of the $C P R S$ gene. By tracking the evolution of the two drug resistance selections with whole genome sequencing, we further demonstrate that the CPRS locus accounts for the majority of genetic adaptation to halofuginone in P. falciparum. We further validate that copy-number variations at the CPRS locus also contribute to halofuginone resistance.

Conclusions: We provide a three-step model for multi-locus evolution of halofuginone drug resistance in $P$. falciparum. Informed by genomic approaches, our results provide the first comprehensive view of the evolutionary trajectory malaria parasites take to achieve drug resistance. Our understanding of the multiple genetic and non-genetic mechanisms of drug resistance informs how we will design and pair future anti-malarials for clinical use.
\end{abstract}

\section{Background}

Malaria is a treatable communicable disease yet remains a common cause of death and disease especially among pregnant women and children. Most of malaria's worldwide burden disproportionately lies in Southeast Asia and SubSaharan Africa, causing over 1.2 million deaths in 2010 [1]. Western medicine's 100+ year history of combating Plasmodium falciparum has taught us that the global population of malaria parasites has a unique and dangerous ability to rapidly evolve and spread drug resistance. Recently it was documented that resistance to the first-line antimalarial artemisinin may be developing in Southeast Asia and the

\footnotetext{
* Correspondence: dfwirth@hsph.harvard.edu

'Department of Immunology and Infectious Disease, Harvard School of Public Health, Boston, MA 02115, USA

${ }^{2}$ Broad Institute of MIT and Harvard, Cambridge, MA 02142, USA

Full list of author information is available at the end of the article
}

molecular underpinnings of artemisinin delayed clearance are begging to be characterized $[2,3]$.

Consequently, it is essential to find new families of antimalarial molecules to take over if artemisinin and its combination-based therapies continue to lose efficacy. Forward genetic drug resistance screening and genomic analysis have previously been used to identify new targets for drug development and understand new drug resistance mechanisms [4-9]. The targets of more than 12 families of small molecules (reviewed in [10]) have been identified in Plasmodia through in vitro selection and genomic characterization of the end-points of these selections. This approach inherently assumes a single mechanism of drug resistance and overlooks the temporality of genetic and non-genetic epistasis involved in the complex evolution of drug resistance in a eukaryotic parasite with a genome of approximately 23 megabases 
and roughly 5,500 expressed proteins in the parasites' life cycles [11].

The dynamics of evolution are essential to understand the drug resistance phenotypes that are readily achieved by Darwinian evolution. Studies in bacterial drug resistance have shown that limited pathways are available due to epistatic interactions between cis- and trans-interacting genetic alterations [12]. Epistasis among genes strongly shape the evolution of microbes [13-15] and viruses [16-18]. Wholegenome whole-population sequencing in bacterial [19] and yeast $[20,21]$ laboratory evolution experiments have proven informative of the evolutionary dynamics at play in longterm adaptation to a variety of selective pressures.

We chose to use a whole-genome whole-population sequencing approach to track the evolutionary dynamics of resistance to the $P$. falciparum cytoplasmic proline tRNA synthetase ( $C P R S$ ) inhibitor halofuginone. tRNA synthetases are very promising anti-parasitic targets for the development novel antimalarials [22] and recent work has found small molecule antimalarials that target the isoleucine tRNA synthetase [23], lysine tRNA synthetase [7], threonine tRNA synthetase [24], and our own work on proline tRNA synthetase (JD Herman et al., submitted). Understanding this interplay of multiple resistance mechanisms is essential for target prioritization, combination therapy design, and drug-resistance surveillance.

\section{Results}

Mutations in the CPRS gene cannot explain evolution of resistance in all long-term selected lines

We previously identified the $c P R S$ gene as the molecular target of halofuginone, and related small molecules (JD Herman et al., submitted). We discovered two unique non-synonymous mutations in the $c P R S$ gene in independent end-point in vitro selection experiments. In this work, we observed that the parasite population gradually acquired resistance to increasing concentrations of halofuginone during the in vitro selection process and we sought to understand this evolution of resistance at a molecular level. Using recent advances in genome sequencing technology and new analytical methods, we characterized two independent selections along their in vitro evolutionary trajectory.

The P. falciparum Dd2 lines halofuginone resistance selected line II (HFGRII) and halofuginone resistance selected line II (HFGRIII) were selected in parallel with an intermittent stepwise strong-selective pressure protocol. Selections began with 10x the parental EC50 for halofuginone $(7 \mathrm{nM})$ and were increased stepwise upon growth tolerance (Additional files 1, 2, and 3). Both HFGRII and HFGRIII grew tolerant of $7 \mathrm{nM}$ halofuginone in 18 generations, 21 $\mathrm{nM}$ in 9 generations, $42 \mathrm{nM}$ in 7 and 9 generations, respectively, and $140 \mathrm{nM}$ in 16 and 22.5 generations, respectively. To confirm these phenotypes, we measured halofuginone dose-response of HFGRII in standard growth assays at selected time points (Figure 1). Consistent with the bulk population growth, HFGRII displayed a constant response to increasing selective pressure.

\section{Genomic mutations only appear after the onset of drug resistance}

We performed whole genome sequencing of the entire evolving population to track the rise and fall of mutant alleles over 50 generations (HFGRII) and 58.5 generations (HFGRIII) (displayed in Additional files 1 and 2). We used our time-course data from HFGRII and HFGRIII to distinguish true mutations from sequencing or alignment-introduced error (filtering scheme described in Additional file 4). Since we were interested in de novo mutational adaptation and our selections began with clonal strains, the two independent replicate populations should not share the exact same mutations. Second, the frequencies of a real mutation should correlate through time (positive autocorrelation), while sequencing errors should be uncorrelated at different time points (negative or zero autocorrelation). We used both de novo mutation and autocorrelation to identify the segregating SNPs and small indels in the independent populations.

From this analysis framework, we found a paucity of genomic mutations over time (Additional file 5). Most of the genetic changes during our evolution experiments occurred at the $C P R S$ locus. With this method, we confirmed the evolution of the C1444T (L482F) cPRS mutation in HFGRII. The C1444T mutation first appeared in the 27th generation at a $0.6 \%$ allele frequency (Figure $2 \mathrm{~A}$ ). However, the $c P R S$

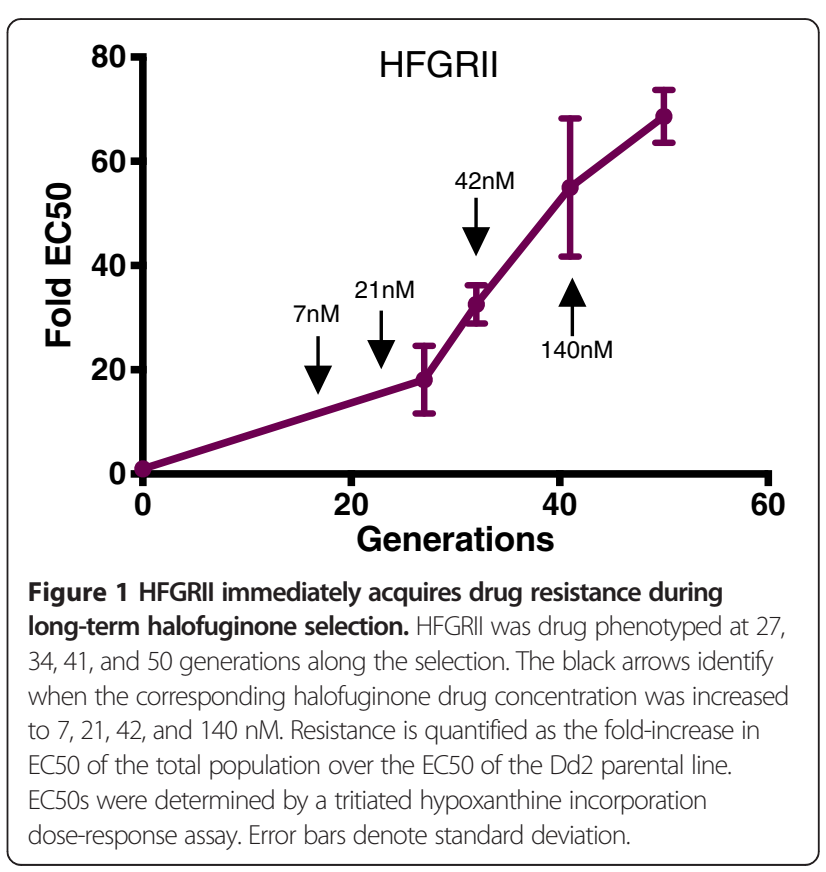




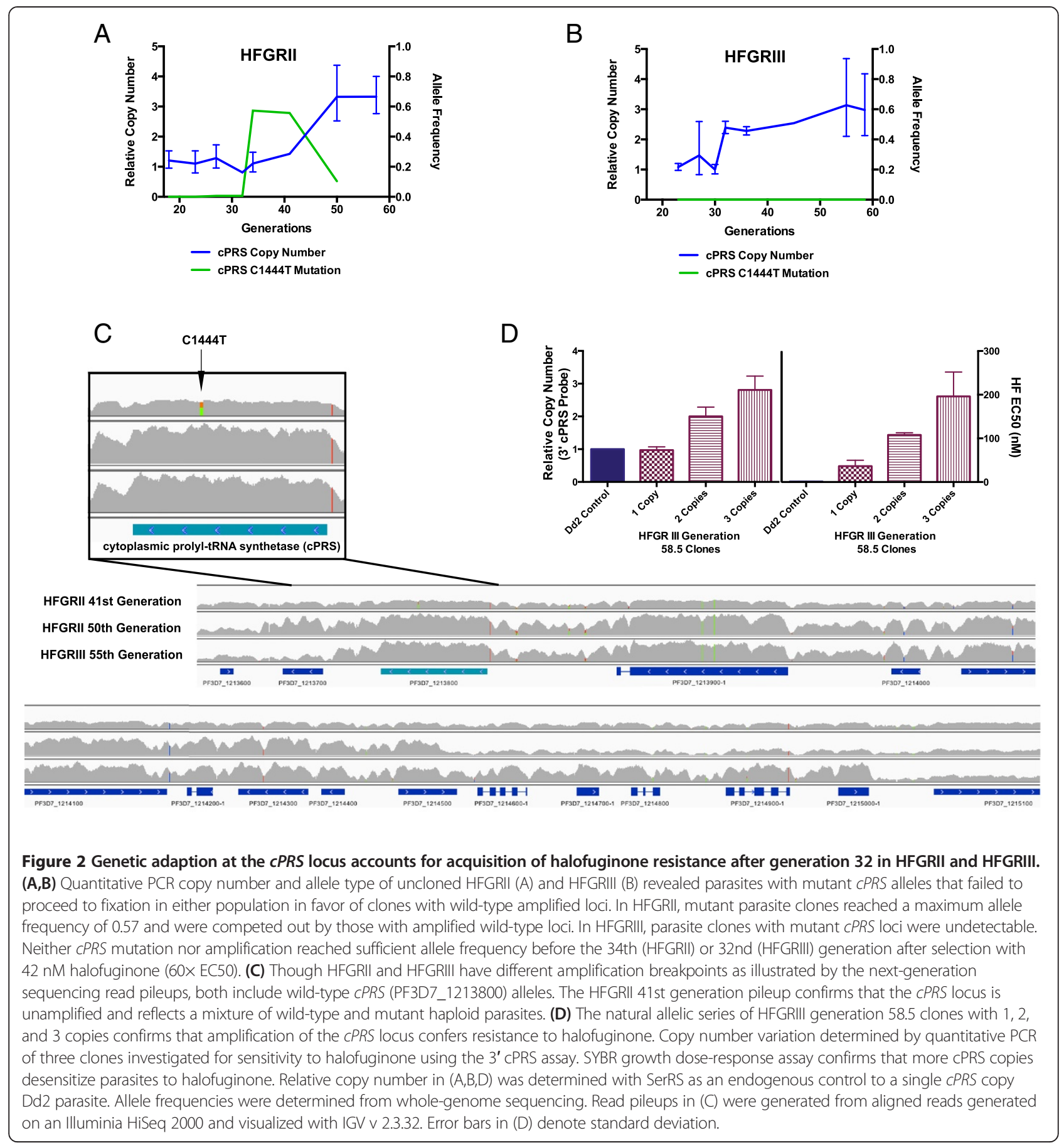

mutation in HFGRII did not proceed to fixation. It reached a maximum allele frequency of $57 \%$, then after 20 additional generations fell from the population.

To supplement the Genome Analysis Toolkit (GA TK)-based analysis of small indels, we searched the pileups from whole-genome sequenced populations for long-scale amplification. We found that the $C P R S$ was part of larger amplifications at later time points in HFGRII and HFGRIII (Figure 2A-C). To better quantify the amplification of $c P R S$ over time in both selections, we performed relative quantification of HFGRII (Figure 2A) and HFGRIII (Figure 2B) using quantitative PCR (qPCR). We found that the $c P R S$ copy number of HFGRII rose as the C1444T $c P R S$ mutation fell in the population. The strong anticorrelation of the two segregating sites implies clonal competition.

Using the same methodologies, we found that no SNPs proceeded to fixation in HFGRIII over the course of the 
experiment (Figure 2B). Rather, in the 32nd generation of the HFGRIII selection, parasites with wild-type (WT) amplified loci first appeared and remained for the duration of the selection. However, from these data we can only determine the average number of $c P R S$ copies across the entire population. To determine the population distribution of parasites with WT amplified $c P R S$ loci, we performed dilutional cloning on the 55th generation of HFGRIII. From 14 cloned parasites, we determined that $71 \%$ of the HFGRIII 55th generation had three WT copies and 21\% had two WT copies of the cPRS locus (Additional file 6). To explore the effect that cPRS copy number variation plays in resistance to halofuginone, we choose three parasite clones with 1, 2, or 3 copies of $c P R S$ (Figure 2D). We found that halofuginone resistance increased with the number of copies of $c P R S$ (Figure 2D) and there was no change in the EC50 of 10 other antimalarial compounds we tested (Additional file 7).

From our whole-genome analysis, we identified genetic adaptations at the $c P R S$ locus from generation 32 and beyond, in both in vitro evolution experiments. However, acquisition of halofuginone resistance preceded $c P R S$ amplification or mutations (Figure 1). By cycle 27 in HFGRII, the bulk population had 18-fold decreased sensitivity to halofuginone (Figure 1) that we cannot explain from our genetic analysis. Without mutations sweeping to fixation, early drug resistance might be achieved in these populations by multiple mutations in independent lineages and would have been missed by metagenomic sequencing. However, the conservative mutation rate and few accumulated mutations observed in a similar $P$. falciparum in vitro culture system [25] argue that a nongenetic adaptive mechanism is more likely.

\section{Induced halofuginone resistance - a stable resistance acquired in eight generations}

We sought to understand the early phase of acquired resistance that was independent of any genetic element we could identify. To probe the early genetic or non-genetic mechanisms of halofuginone resistance, we created the halofuginone-induced resistance model. We put clonal WT Dd2 parasites under constant low halofuginone pressure $(2.8 \mathrm{nM})$ five-fold lower than that used in the first step of the selection of HFGRII and HFGRIII. At first, the parasite populations decreased below the detection limit of thin smear microscopy. Within eight to nine generations, both replicate-treated $\mathrm{Dd} 2$ lines recrudesced and were more than 20-fold resistant compared with an untreated Dd2 line (Figure 3). This induced resistance was stable and heritable in both Dd2 biological replicates, Dd2 Induced 1 and Dd2 Induced 2, for greater than 30 generations without drug pressure. We also replicated these experiments in the unrelated parasite line HB3 (Additional

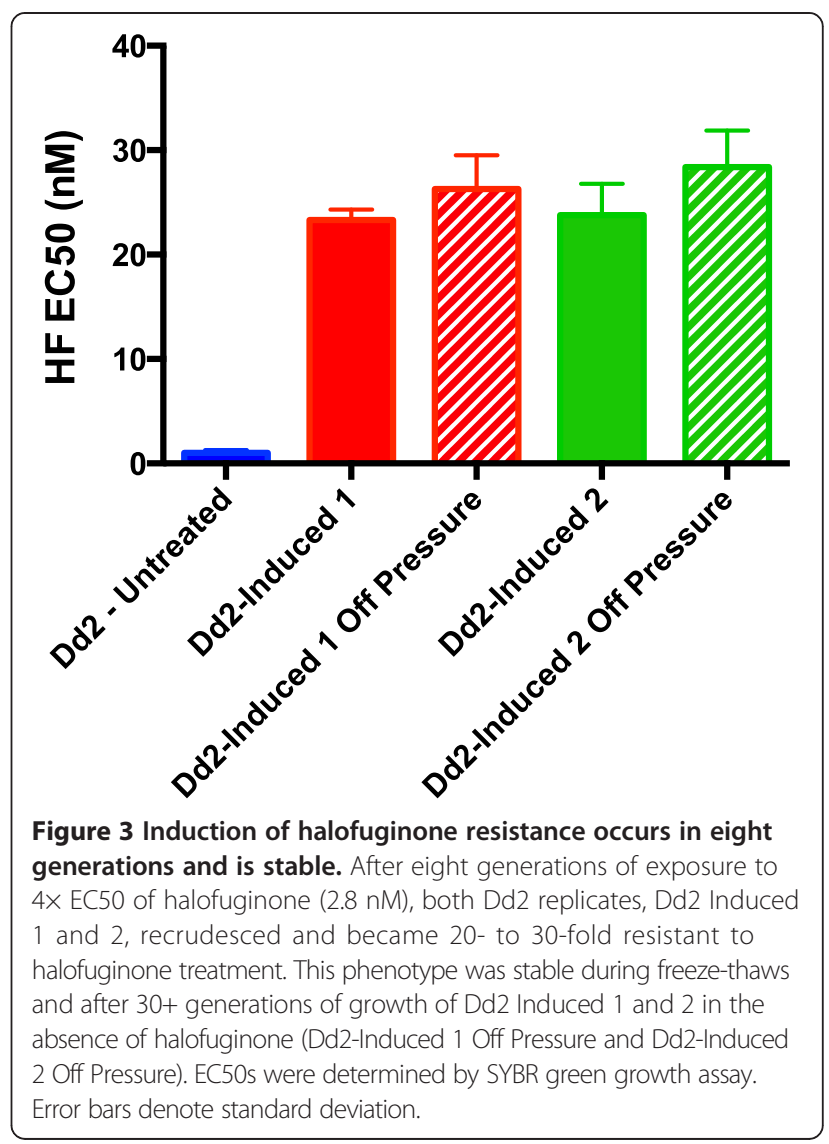

file 8). First we validated that Dd2 Induced 1 and 2 had no mutations or copy number variations at the $c P R S$ locus (Additional file 9). Next, we performed whole-genome sequencing on the parental strain and Dd2 Induced 1 and 2. Of the five genes with non-synonymous SNPs called between the parental and induced strains, four were eliminated as alignment/SNP-calling error with Sanger sequencing (Additional file 10) and a fifth was not found in replicate inductions. Thus, we concluded sweeping genetic adaptation could not account for the halofuginone induction phenotype.

\section{Increased proline concentration results in abrupt induction of halofuginone resistance}

We wanted to understand the mechanism of induced resistance and therefore investigated amino acid homeostasis in our induced parasites. Proline is the only of cPRS's three substrates (ATP, proline, and uncharged proline tRNA) that we believe competes with halofuginone for the cPRS binding pocket based on structural studies (JD Herman et al., submitted) [26,27]. We investigated whether parasites with halofuginone-induced resistance have altered amino acid homeostasis using a liquid crystallography-mass spectrometry (LC-MS) metabolomics approach. 
We measured 19 intracellular amino acid levels in our Dd2 Induced 1 and Dd2 Induced 2 parasites and found proline to be uniquely upregulated. We examined the metabolomics picture of these parasites by measuring both saponin-freed P. falciparum (Figure 4) and P. falciparum infected red blood cells (iRBCs) (Figure S4a in Additional file 11). This provided further insight into the dynamics of proline accumulation that may contribute to induced halofuginone resistance. Both free parasites and total iRBCs had increased proline concentrations when compared with the parental Dd2 line (Figure 4A; Additional file 11). The free parasite fraction had a 19- to 32-fold increase while the total iRBC sample had a 3- to 5-fold increase, consistent with the parasite cytosol as the source of metabolic enrichment. To validate that this is a cell line autonomous phenomenon, we tested and found proline-specific upregulation in halofuginone-induced HB3 parasites as well (Figure S5b,c in Additional file 8).

\section{Increases in proline are strongly associated with halofuginone resistance}

To understand the initial timing and subsequent dynamics of induced halofuginone resistance associated with increased proline, we measured intracellular proline concentration immediately after recrudescence and in subsequent generations of Dd2 Induced parasites. We found that proline concentrations were increased upon our first technically feasible measurement and did not vary significantly up to 60 generations after, while parasites were maintained under constant halofuginone pressure (Figure S6a in Additional file 12).
Further, we wanted to understand if the dynamics of increased proline in the Dd2 Induced parasites corresponded with increased halofuginone resistance. We found that the increase in the halofuginone dose-response was seen as early as the fifth generation post-recrudescence. While maintained on $4 \times$ EC50 halofuginone pressure, halofuginone resistance in Dd2 Induced parasites remained constant over 60 generations (Figure S6b in Additional file 12) as did proline levels (Figure S6a in Additional file 12).

Further, we wanted to understand if increased proline autonomous of any cell-intrinsic adaptation could change the dose-response of halofuginone. To this end, we measured the dose-response of halofuginone in untreated $\mathrm{Dd} 2$ parasites growing in media with a gradient of proline concentrations in a 72-hour assay. We found that increasing proline concentrations in the growth media can give $P$. falciparum an apparent resistance to halofuginone (Additional file 13) without any pre-conditioning.

\section{New proline homeostatic set-point is stable}

Next we wanted to determine the relevance and permanence of elevated intracellular proline as a resistance mechanism in P. falciparum. To test the stability of this proline enrichment, we took $\mathrm{Dd} 2$ Induced parasites grown without halofuginone pressure for more than 30 generations (Figure 3) and measured their amino acid levels. We found that both biological replicate Dd2 inductions maintained their drug resistance and their specific upregulation of proline (Figure 4).
A

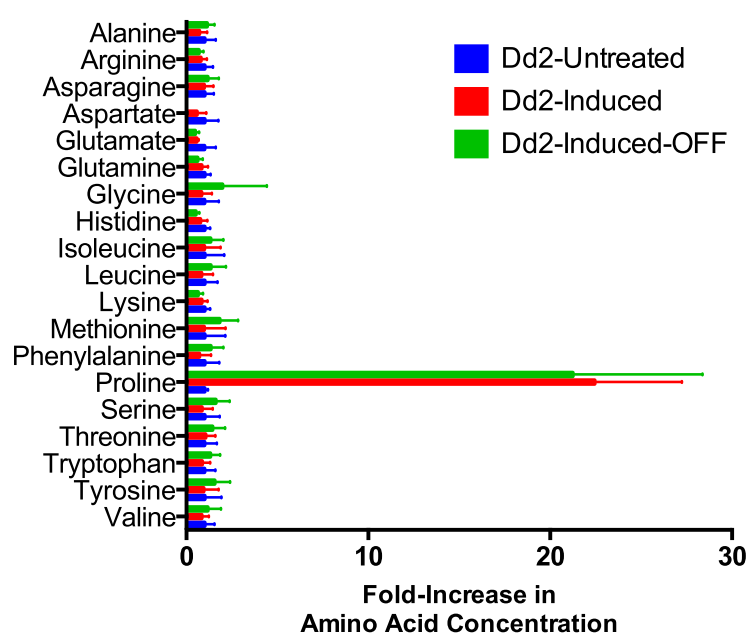

\section{B}

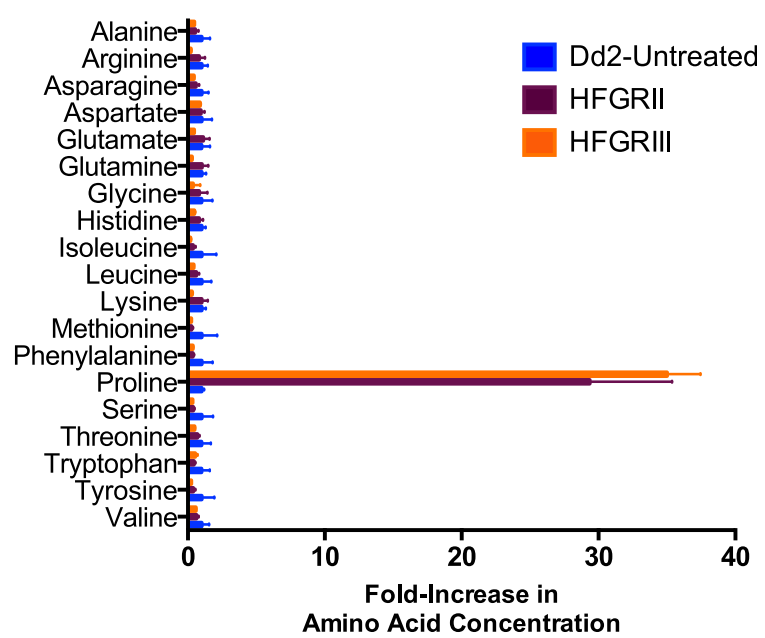

Figure 4 Halofuginone short-term induced and long-term selected parasites specifically upregulate intracellular proline concentration. (A,B) Of the 19 proteogenic amino acids assayed from saponin-released purified parasites, only proline concentrations were increased in Dd2 Induced parasites (A) and long-term selected lines HFGRII and HFGRIII (B). The fold increase in amino acid concentration upon induction or selection is expressed as a ratio of induced/selected line measurement over parental Dd2 line measurement. Amino acid levels of saponin-released $P$. falciparum parasites were quantified from the normalized integrated peak intensity determined by LC-MS. Error bars denote standard deviation. 
To understand whether increased intracellular proline is a shared mechanism of resistance between our short-term induced and long-term halofuginone selected parasites, we measured the amino acid levels of HFGRII and HFGRIII parasites. We found that long-term selected parasites have uniquely elevated proline similar to halofuginone-induced parasites (Figure 4B; Figure S4b in Additional file 11). This further confirmed the stability of the proline-related upregulation and showed that two long-term selected lines, HFGRII and HFGRIII, also use this halofuginone drugresistance mechanism.

\section{Proline-like non-proteogenic amino acids are also upregulated during halofuginone-induced resistance}

To better understand the specificity of amino acid reregulation halofuginone induces, we examined the concentration of 96 additional polar metabolites (Additional file 14) in halofuginone short-term induced and long-term selected parasites. We found two metabolites whose parasite intracellular concentration was highly correlated with proline: cis/ trans-hydroxyproline and pipecolic acid (homoproline) (Figure 5A,B). These strong correlations were present when limiting analysis to HB3 untreated and induced parasites, Dd2 untreated and HFGRII and HFGRIII parasites, and also Dd2 untreated and induced parasites (Figure 5A). Pipecolic acid, a proline-like non-proteogenic amino acid, is known to be formed by the degradation of lysine and not proline [28]. We also found that hydroxyproline and pipecolic acid were similarly enriched in all parasites with elevated intracellular proline while lysine levels were unchanged (Figure 5C). Though non-enzymatic oxidation of proline could produce hydroxyproline that would be highly correlated with proline levels, pipecolic acid could not be derived from proline non-enzymatically. Additionally, levels of lysine, the metabolic precursor of pipecolic acid, are unperturbed in halofuginone-induced parasites, suggesting a primary enrichment of pipecolic itself (Figure 5C).

\section{Halofuginone-induced resistance is not associated with changes in gene expression}

The enrichment of proline and proline-like amino acids directed our interest to clag 2 and 3.2 - two members of a multigenic family implicated in the increased flux of proline across the iRBC membrane via the plasmodiumspecific anion current [29-32]. After the publication of two papers supporting the involvement of clag genes in the acquisition of resistance to the antimalarial blasticidin $[33,34]$, we became interested in whether a similar epigenetic mechanism explained the rapid acquisition of halofuginone resistance. We found that clag 2 and 3.2 were not consistently differentially expressed in halofuginone-induced parasites (Figure S9a in Additional file 15). Their pattern of expression varied most consistently based on their maturity within the early schizont stage (approximately 34 hours postinfection (hpi)) rather than between untreated and halofuginone-induced parasites.

We also took a genome-wide approach to evaluate changes in gene expression that could explain the nongenetic resistance to halofuginone and would lead to an epigenetic mechanism. The clag family of genes is one of the few examples of $P$. falciparum genes that show steadystate differences in RNA transcript levels in response to external stimuli [33,34]. Steady-state RNA transcript abundance is hard wired to the $P$. falciparum life cycle [35]. We performed RNA-Seq differential expression analysis between parasites with non-genetic halofuginone drug resistance and their parental Dd2 strain.

To disentangle stage-specific from phenotypic differential expression, we compared our RNA-Seq expression data with an RNA-Seq time course with $4 \mathrm{~h}$ resolution (KM Broadbent et al., submitted). We determined which stage in the IDC time course each of our RNA-Seq libraries most closely resembled (Figure S9b in Additional file 15); the untreated Dd2 library most strongly correlated with the 34 hpi time point while the Dd2-Induced 1 and Dd2-Induced 2 libraries most strongly correlated with the 38 hpi time point. Next, we performed in silico differential expression analysis to generate a list of genes differentially expressed based on the variation in stage inherent in P. falciparum experimentation. By creating a linear fit between the stage controls, from the time course, and our halofuginoneinduced parasites (Figure S9c in Additional file 15), we eliminated stage-specific artifacts and were left with a list of seven differentially expressed genes (Figure S9d in Additional file 15). These differentially expressed genes represent a mixture of genes differentially expressed between Dd2 and the 3D7 parasite used for the control time course and genes potentially involved in halofuginoneinduced resistance.

\section{Discussion and conclusions}

We previously identified the cPRS as the target of febrifugine derivatives such as halofuginone and halofuginol in $P$. falciparum (JD Herman et al., submitted). In the present study, we took a genomic evolution approach using metagenomic sequencing to investigate the temporal emergence of drug resistance and discovered a novel non-genetic adaptation that occurs rapidly upon exposure to halofuginone. This precedes either SNP emergence in $C P R S$ or amplification of the $C P R S$ locus, both of which occur after prolonged, stepwise selection. By profiling polar metabolites, we found that re-calibrating proline homeostasis is central to halofuginone short-term induced resistance and a novel interplay of non-genetic and genetic mechanisms contributing to malaria drug resistance.

Whole genome sequencing of HFGRII at different time points has provided deep insight into the relative fitness of 


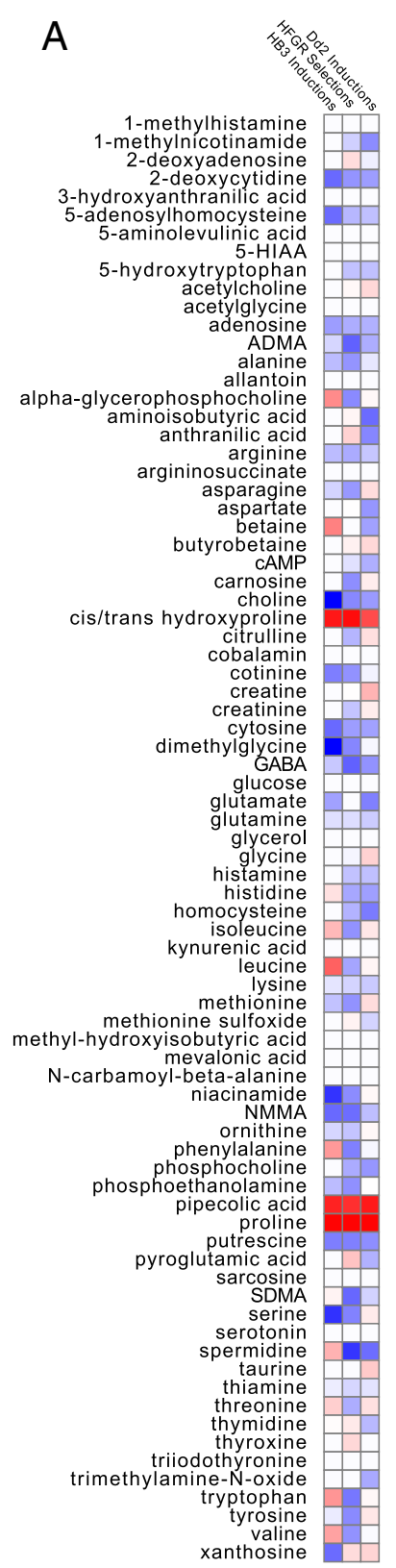

B<smiles>O=C(O)C1CCCN1</smiles>

$\mathrm{HO}$,

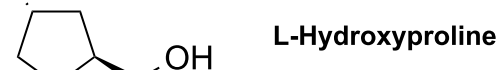

C

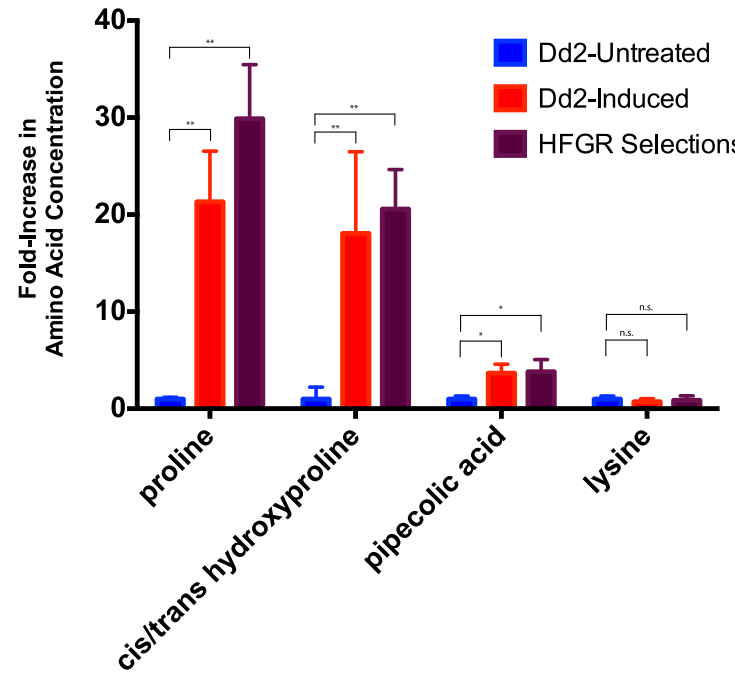

Pipecolic acid $\mathrm{H}$<smiles>O=C(O)C1CCCCN1</smiles>

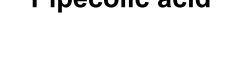

Figure 5 Profiling of 115 polar metabolites reveals that proline and proline-like non-proteogenic amino acids are uniquely enriched in halofuginone short-term induced and long-term selected parasites. (A) Of the 115 polar metabolites measured, only pipecolic acid and cis/ trans-hydroxyproline concentrations are highly correlated with cytosolic proline concentrations in short-term induced Dd2 parasites, short-term induced HB3 parasites, and long-term selected Dd2 lines HFGRI, II, and III. Each column represents an independent dataset analyzed: HB3 untreated and induced parasites; Dd2 untreated and HFGRII and HFGRIII parasites; and also Dd2 untreated and induced parasites. The full metabolite profile is provided in Additional file 14. (B) The three amino acids all contain cyclic secondary amines and carboxylate groups. The addition of a hydroxyl and replacement of a 5-member for a 6-cyclic amine result in the slightly different chemical and physical properties between proline and hydroxyproline and pipecolic acid, respectively. (C) Cytosolic proline, cis/trans-hydroxyproline, and pipecolic acid are elevated in halofuginone-resistant parasites compared with an untreated Dd2 line. The cytosolic concentration of the pipecolic acid precursor lysine is unaffected. The heat map in (A) represents the correlation coefficients (r-values) between cytosolic concentrations of metabolites with that of proline in three collections of WT and modified parasites. High correlation $(r=1)$ is represented by red while low correlation $(r=-1)$ is represented by blue. Statistical significance in $(C)$ was determined with multiple unpaired $t$-tests using the Sidak-Bonferroni method, with alpha $=0.05$. Significant results are indicated by asterisks: single asterisks indicate uncorrected $P<10^{-7}$ and double asterisks indicates uncorrected $P<10^{-10}$. Error bars denote standard deviation. 
cPRS mutations and amplification. By looking at snap shots of our in vitro evolution experiments, we saw the rise and fall of $c P R S$ mutant and WT alleles. The clonal competition between WT amplified $c P R S$ and C1444T cPRS single-copy clones observed between the 41st and 58th generation argues that $c P R S$ amplifications are more fit at higher halofuginone drug pressures than the HFGRII cPRS mutants. As a pulsed-drug in vitro selection, the advantage of amplified $c P R S$ alleles represents clonal competition in the presence and absence of halofuginone. In the light of our findings in JD Herman et al., (submitted) that cPRS mutant proteins have decreased affinity for their substrate proline, our genomic approach further supports our suspicion that $c P R S$ mutations confer a competitive fitness cost and validates targeting $C P R S$ as a viable and promising strategy for the development of future antimalarials.

Using our induced resistance parasite lines, we sought to understand the underlying non-genetic mechanism of resistance to halofuginone. We found that proline was increased 20 - to 30 -fold in the cytoplasm of $P$. falciparum parasites with short-term induced resistance (Figure 3; Figure S5a in Additional file 8). The pattern of proline and proline-related amino acid transport (Figure 5C) is highly similar to that performed by the high-affinity mammalian neuronal Lproline transporter SLC6A7 (PROT) present in gabaergic synapses [36] and epithelial L-proline transporter SLC6A20 (IMINO) [37]; in fact characterization of hPROT found pipecolic acid was the strongest competitive inhibitor of Lproline transport among six analogues [36]. These data are highly suggestive of a proline-specific importation mechanism underlying halofuginone-induced resistance. We are currently investigating P. falciparum SLC6A homologues for their role in proline transport and halofuginone-induced resistance. We have determined that there are no genic mutations in the halofuginone long-term selected or short-term induced parasites in the SLC6A homologues. Alternative explanations include reduced catabolism of proline to arginine [38] or alterations in proline export, as has been reported in prokaryotes [39].

Thus, our metabolomics analysis is highly suggestive that non-genetic halofuginone resistance is caused by a proline-specific transport mechanism. Comparison of our steady-state measurements of total iRBCs and purified parasites implies that this transporter is located on the parasite or parasitophorous vacuolar membrane. However, further experimental work will be required to confirm this hypothesis.

The implication that proline transport is involved in halofuginone-induced resistance led us to investigate the involvement of the clag multi-gene family. We see no cell cycle-independent changes in clag gene expression in qPCR or RNA-Seq studies (Additional file 15). Our stagecontrolled RNA-Seq analysis revealed seven differentially expressed genes. However, we cannot determine whether they represent genes involved in non-genetic halofuginone resistance or genes differentially expressed between the $P$. falciparum Dd2 and 3D7 strains. None have clear involvement in proline biogenesis, transport, or protein degradation. Additionally, none are homologous to the SLC6A family of amino acid transporters. Thus, we have not found strong evidence that differential RNA expression can explain non-genetic halofuginone resistance.

We believe the stable upregulation of intracellular amino acid homeostasis represents a new and potentially general mechanism of drug resistance in malaria. The non-genetic alteration of proline amino acid transport is a strainindependent stable mechanism of halofuginone resistance. We as a field have focused on identifying genetic mechanisms of malarial drug resistance with the assumption that they are the primary contributors to stable and heritable resistance. Our evidence of an in vitro stable non-genetic mechanism of resistance questions this assumption. Though the alteration of intracellular proline we found will likely not be a pan-drug resistance mechanism, it suggests that metabolic mechanisms of drug resistance/tolerance exist in $P$. falciparum. The drug resistance literature in the field of cancer biology has also begun to appreciate metabolic methods of drug resistance. Changes in central carbon metabolism [39], including the Warburg effect [40], and in amino acids involved in oxidative stress responses [41,42] have been demonstrated to confer drug resistance in tumor cells. Combining proteomic and metabolomic approaches will be essential to understanding the metabolomic basis of malaria drug resistance.

The non-genetic proline-based resistance we have found in halofuginone-induced parasites differs from previous instigations of amino acid regulation in $P$. falciparum. Much previous work in P. falciparum amino acid homeostasis has focused on the partially conserved amino acid starvation pathway $[23,43,44]$, which results in translational inhibition and a hibernation-like state [44]. We believe that this stable non-genetic resistance is not an immediate starvation response, but a reregulation of metabolism. Our group has also shown that halofuginone activates the amino acid starvation pathway within 90 minutes (JD Herman et al., submitted). Unlike the amino acid starvation pathway, in which signal transduction occurs within minutes to hours, halofuginone induction of increased intracellular proline occurs after multiple generations of growth. In addition to the temporality, the two biological phenomena differ in their effect on intracellular amino acid levels. Babbit et al. [44] saw no difference in either proline or isoleucine levels between isoleucine-fed and isoleucinestarved parasites. Based on temporality and amino acid homeostatic concentrations, we thus posit that halofuginone-induced resistance represents a separate 
biological phenomenon resulting in long-term upregulation of proline homeostasis.

To explain the multistep mechanisms of halofuginone drug resistance, we propose a multi-step adaptation process that maximizes fitness in increasing concentrations of halofuginone. In the first phase of our in vitro evolution, resistance is acquired by altering cellular amino acid homeostasis; specifically, intracellular proline levels are elevated in response to halofuginone pressure. This phenomenon is analogous to the induced resistance phenotype we found with low-dose constant drug treatment. The second phase of drug resistance represents alterations in $C P R S$ in mutually exclusive ways. We have witnessed either target-site mutations or amplification in this second temporal phase. However, the third phase of drug resistance encompasses amplification of the WT target loci in the presence of increasing selective drug pressure regardless of prior mutation (Figure 6). This result implies that a mutation in $c P R S$ renders the parasite less fit. Our attempts at allelic replacement of the WT genomic copy with a single copy of the C1444T cPRS allele have been unsuccessful; all clones that have been isolated after transfection contain multiple copies of the $c P R S$ gene with both WT and mutant alleles present and all have elevated intracellular proline levels (data not shown).

Our understanding of the sequential acquisition of halofuginone resistance is contrary to the commonly used model of compensatory mutations. In such a model, the evolution of drug resistance results in organisms with decreased fitness in the absence of drug pressure $[45,46]$. Additional 'compensatory' mutations are acquired to rescue the fitness of the drug-resistant organism. Rather than finding post-cPRS compensatory mutations, we found that metabolic cellular adaptations preexisted $c P R S$ mutations under halofuginone selective pressure. This model has larger implications for understanding the initial steps of evolutionary adaptation.
Mapping resistance mechanisms is also essential to furthering malarial drug discovery. Identifying resistance mechanisms will inform the future usefulness of an antimalarial compound. Further, this model of halofuginone resistance will inform the rational design of combination therapy, most obvious of which is the combination of febrifugine derivatives with inhibitors to the yet-to-be identified proline transport mechanism. With this combination, we could block the early non-genetic adaption that precedes the evolution of genetic resistance. Thus, rather than targeting the resistant enzyme and making it less fit [47], one could propose a strategy to eliminate the evolutionary path that would lead to target site mutations and amplification at the $C P R S$ locus.

Our whole-genome population sequencing approach revealed a non-genetic stable resistance that we have tracked to amino acid homeostasis. We believe genomic technologies allow exploration beyond the obvious next step hypothesis and unlock a powerful new level of insight and progress for the field of malariology and infectious disease research in general.

\section{Materials and methods}

Metagenomic whole-genome sequencing of halofuginone populations selected in vitro

The goal of this experiment was to define the evolutionary trajectory of halofuginone drug resistance in a P. falciparum in vitro long-term culture experiment. We performed two replicate long-term intermittent pressure selections in order to increase the concentration a clonal strain of $\mathrm{Dd} 2$ parasites could tolerate; we named the two strains HFGRII and HFGRIII. We started with $10 \times$ EC50 $(7 \mathrm{nM})$ and subsequently increased the pressure to $30 \times(21 \mathrm{nM}), 60 \times(42$ $\mathrm{nM}$ ), and then $200 \times(140 \mathrm{nM}$ ) (Additional files 1, 2, and 3). The notation HFGRII 2-10x signifies the selection HFGRII was selected two times with $10 \times$ EC50 $(7 \mathrm{nM})$. At each step along the selection, we isolated genomic DNA from the entire evolving population. We chose six time points for

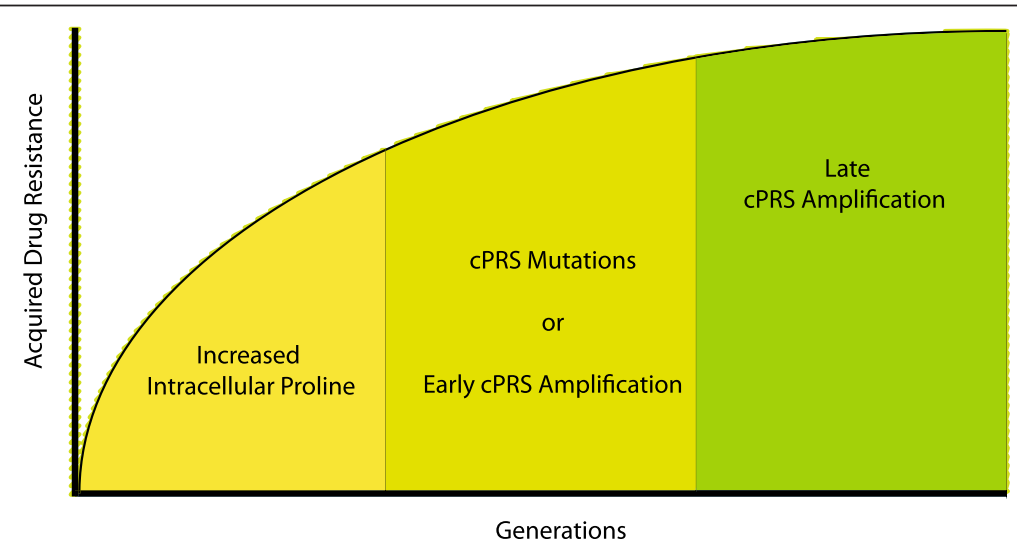

Figure 6 A model of step-wise acquisition of halofuginone drug resistance. 
HFGRII and seven time points for HFGRIII spaced across the approximately 60 generations of halofuginone selection in order to track the multiple genetic mechanisms that may contribute to halofuginone resistance.

Genomic DNA extracted from bulk population cultures of the two independent lines selected for halofuginone resistance was extracted and sheared with a Covaris S220 Focused-ultrasonicator (Covaris, Woburn, MA, USA). Illumina-compatible libraries were prepared on the Apollo 324 (WaferGen Biosystems, Fremont, CA, USA) and sequenced on an Illumina HiSeq 2000 (Illumina, San Diego, CA, USA). P. falciparum populations were sequenced with the goal of reaching over $60 \times$ average fold-coverage across the genome.

\section{Metagenomic time series analysis to identify segregating sites}

We used a two-stage process to distinguish real mutations from sequencing and alignment errors in the metapopulation time-series sequence data. First, we used the GATK (Broad Institute, Cambridge, MA, USA) to obtain a permissive list of candidate loci and the number of supporting reads for each allele at each timepoint. We used the haploid mode of GATK's Unified Genotyper with a minimum confidence threshold phred score of 4 to obtain a permissive set of SNPs and small indels. We further filtered this set of candidates based on two fundamental assumptions: first, the two independent replicate populations should not share exactly the same mutations; and second, the frequencies of a real mutation should be correlated through time (positive autocorrelation), while sequencing errors should be uncorrelated at different time-points (negative or zero autocorrelation).

Thus, we 1) discarded all sites with an average coverage depth less than $10 \times$ across the time-points or no coverage at more than three time-points, 2) discarded all sites with an average frequency above $1 \%$ in the non-focal population, 3) discarded all sites with a negative or zero autocorrelation, and 4) discarded all mutations supported by fewer than 10 total reads or with a maximum frequency of less than $10 \%$. Further, we noticed that the frequencies of alternative alleles in HFGRII at time-point 2-30× (27th generation) were aberrant despite deep coverage and we discarded this time-point from the analysis due to potential contamination.

\section{Metagenomic time series analysis to identify selective sweeps}

We took the unfiltered list of candidate loci called by the Unified Genotyper (GATK) and looked for mutations that began at a frequency near zero at the first time-point and ended at a frequency near one at the last time-point.

\section{Metagenomic time series analysis to identify hitchhiking mutations along with CPRS mutation in HFGRII}

We searched for mutations that hitchhiked with the known cPRS mutation C1444T in HFGRII. To find hitchhiking mutations, we sorted the unfiltered list of candidate loci called by the Unified Genotyper (GATK) by the Euclidean distance between their allele frequency trajectories and the frequency trajectory of the focal mutation.

\section{Quantitative PCR - relative copy number analysis}

Genomic DNA was prepared from saponin-lysed P. falciparum parasites using the Qiagen Blood Mini- or Midi Kits (Qiagen, Venlo, Limburg, the Netherlands). qPCR analysis was performed on an Agilent 7900HT Fast Real-Time System (Agilent Technologies, Santa Clara, CA, USA) using unlabeled primers and Power SYBR Green master mix. Primers used for copy number analysis are listed in Additional file 16 (control locus primers) and Additional file 17 (target locus primers). Copy number was calculated using the $\Delta \Delta C$ t method included in SDS version 2.3.2 as described in the Applied Biosystems User Bulletin 2.

\section{In vitro drug sensitivity and dose-response analysis by SYBR Green staining}

The SYBR Green I method was used as previously described [48]. In brief, we grew $P$. falciparum parasites for 72 hours in 384-well plates at 1\% hematocrit and 1\% starting parasitemia. Growth was assessed by SYBR Green staining of parasite DNA. All dose-response assays were carried out with 12-point curves in technical triplicate. DMSO stocks of drugs used were dispensed by HP D300 Digital Dispenser (Hewlet Packard, Palo Alto, CA, USA). Fluorescence measurements were collected on a SpectraMax M5 (Molecular Devices, Sunnyvale, CA, USA) and analyzed on GraphPad Prism version 5 (GraphPad Software, La Jolla, CA, USA) and EC50 values were determined with the curve-fitting algorithm Log(inhibitor) versus Response - Variable slope.

\section{In vitro drug sensitivity and dose-response analysis by tritiated hypoxanthine assay}

Halofuginone dose-response assays were performed as described by Desjardins et al. [49] using the initial drug resistance selected HFGRII parasites.

\section{Parasite culture}

Malaria culture was performed as described by Trager and Jensen [50]. Dd2 is a chloroquine-resistant in vitro cultured parasite cloned from the Laos derived W2-MEF parasite obtained from MR4 (ATCC, Manassas, VA, USA). 


\section{Flow cytometry}

Flow cytometry of P. falciparum-infected erythrocytes was carried out based on SYBR Green I staining of parasite nuclei as previously described [51]. Modifications to the Bei et al. [51] protocol include staining of iRBCs with SYBR Green I at a 1:2,000 concentration, and acquisition of non-single cells pre-filtered for cells that fell on the $\mathrm{y}=\mathrm{x}$ line in a plot of forward scatter area versus height. All flow cytometry was collected on a MACSQuant Flow Cytometer (Miltneyi Biotec Inc., San Diego, CA, USA) and analyzed with MacQuantify and FlowJo 8.8.6 software (Tree Star, Ashland, OR, USA).

\section{LC-MS analysis of amino acids and polar metabolites}

Highly synchronous (within 4 hours) early schizonts were magnetically purified with MACS CS columns (Miltneyi Biotec Inc., San Diego, CA, USA). A small aliquot was also made for flow cytometry. The rest of the purified samples were divided into two equal volumes: one for saponin lysis $(0.025 \%)$ and one for whole iRBC extraction. Each sample was washed twice in phosphatebuffered saline, and then suspended in $10 \mu \mathrm{l}$ phosphatebuffered saline (Life Technologies, Carlsbad, CA, USA). Polar metabolites were extracted using nine volumes of 74.9:24.9:0.2 (v/v/v) acetonitrile/methanol/formic acid containing stable isotope-labeled internal standards $(0.2 \mathrm{ng} / \mu \mathrm{l}$ valine-d8 (Sigma Aldrich, St. Louis, MO, USA); and $0.2 \mathrm{ng} / \mu \mathrm{l}$ phenylalanine- $\mathrm{d} 8$ (Cambridge Isotope Laboratories,Tewksbury, MA, USA)). Profiles of amino acids were measured using LC-MS as described previously [52]. Briefly, positive ionization, multiple reaction mode (MRM) data were acquired using a 4000 QTRAP triple quadrupole mass spectrometer (AB SCIEX, Framingham, MA, USA) coupled to an 1100 Series pump (Agilent) and an HTS PAL autosampler (Leap Technologies, Carrboro, NC, USA). Cell extracts $(10 \mu \mathrm{l})$ were injected onto a $150 \times 2.1 \mathrm{~mm}$ Atlantis HILIC column (Waters, Milford, MA, USA). The column was eluted isocratically at a flow rate of $250 \mu \mathrm{l} / \mathrm{mi}$ nute with $5 \%$ mobile phase A $(10 \mathrm{mM}$ ammonium formate and $0.1 \%$ formic acid in water) for 1 minute followed by a linear gradient to $40 \%$ mobile phase B (acetonitrile with $0.1 \%$ formic acid) over 10 minutes. The ion spray voltage was $4.5 \mathrm{kV}$ and the source temperature was $450^{\circ} \mathrm{C}$. MultiQuant 1.2 software (AB SCIEX) was used for automated peak integration and metabolite peaks were manually reviewed for quality of integration and compared against a known standard to confirm identity. Stable isotope-labeled internal standards were used to eliminate samples with poor data quality. Metabolite peaks signals were total-signal normalized with all 115 metabolites. Pearson correlation analysis was performed in prism and heat maps were generated with Gene-e (Broad Institute).

\section{Quantitative PCR - gene expression analysis}

Total RNA was extracted using Trizol (Life Technologies, Carlsbad, CA, USA) according to the manufacturer's instructions, DNAse-treated, and re-purified with Qiagen RNeasy mini columns. First strand cDNA synthesis was performed using SuperScript III (Life Technologies) following the manufacturer's instructions. The absence of contaminating DNA and the success of the reverse transcriptase reaction were confirmed by comparing qPCR of Rt + and Rt- with the control seryl tRNA synthetase primer set in quadruplicate; samples were run on and ABI $7900 \mathrm{HT}$ and fold expression calculated using the ABI software suite SDS 2.3.2. cDNA concentrations were normalized to SerRS Ct values to minimize biases in PCR efficiency. Samples were run in quadruplicate with two control primer sets for validation of expression analysis. PCR amplification was performed as follows: 15 minutes at $95^{\circ} \mathrm{C}$ followed by 40 cycles of two-step amplification of $94^{\circ} \mathrm{C}$ for $30 \mathrm{~s}$ and $52^{\circ} \mathrm{C}$ for $30 \mathrm{~s}$. All primers used for expression analysis (Additional files 16,17 , and 18) were validated for specificity and efficiency under the same PCR conditions.

\section{RNA-Seq expression analysis}

Synchronized late schizont $P$. falciparum Dd2 untreated and Dd2 Induced 1 and Dd2 Induced 2 parasites were lysed with saponin. Total parasite RNA was purified with Qiagen RNeasy mini columns. The polyA tagged RNA was purified with PrepXTM PolyA Protocol on an Apollo 324 Library Prep System (Wafergen). Strandspecific RNA-Seq libraries were assembled with the PrepX mRNA Library Protocol and quantified with the Kapa NGS Library Quantification Kit. Libraries were sequenced on an Illumina HiSeq 2000 using 101-bp, paired-end read technology.

Raw reads were aligned using TopHat 2.0.1 against the $P$. falciparum 3D7 PlasmoDB version 10.0 genome. Because so many genes in the falciparum genome are homologous, very stringent alignment parameters were used: -r 300 -mate-stddev 100 -library-type fr-firststrand -i 70 -I 5000 -readmismatches 0 -segment-mismatches 0 -max-segment-intron 5000 -max-coverage-intron 5000 -b2-very-sensitive -readgap-length 0 -read-edit-dist 0 -read-realign-edit-dist 0 -maxdeletion-length 0 -max-insertion-length 0 -max-multihits 2 -no-mixed -no-discordant to be consistent with the 3D7 RNA-Seq 4-h time course from Broadbent et al. (submitted). Roughly one-third of reads could be aligned using these settings.

To determine the exact stage of the $\mathrm{Dd} 2$ control and experimental expression profiles, correlation analyses were performed using Python, numpy, scipy.stats.stats, and plotted using matplotlib. Spearman correlation was calculated between each of the three Dd2 RNA-Seq libraries and the nine 3D7 RNA-Seq time-points 
separated throughout the 48-h life cycle (KM Broadbent et al., submitted).

Gene expression was quantified using Cufflinks 2.2, with annotations from PlasmoDB version 10. Differential expression of genes in the Dd2 Induced 1 and Dd2 Induced 2 samples were calculated with respect to the $\mathrm{Dd} 2$ untreated sample. To control for stage-specific differentially expressed genes, we calculated differential expression between the 3D7 time-points that most strongly correlated with the Dd2 induced and Dd2 untreated samples. Distributions of $\log$ transformed fold changes of the $\mathrm{Dd} 2$ and corresponding 3D7 time-points were analyzed using $\mathrm{R}$, and plotted using ggplot2. Outliers from the linear fitting that were shared between the two biological replicate inductions with more than 10 reads per gene model in each RNA-Seq library were determined to be differentially expressed.

\section{Data availability}

BAM files for all genomic and transcriptomic analysis are accessible on NCBI's Sequence Read Archive with BioProject accession ID PRJNA167166.

\section{Additional files}

Additional file 1: Table S1. Sequenced time-points from the HFGRII in vitro evolution experiment.

Additional file 2: Table S2. Sequenced time-points from the HFGRIII in vitro evolution experiment.

Additional file 3: Figure S1. Diagram of long-term drug resistant selection experiments, demonstrating the stepwise intermittent selection protocol used for long-term evolutionary studies of the halofuginone resistance trajectory in HFGRII and HFGRIII.

Additional file 4: Table S3. Filtering scheme for metagenomic sequence analysis of HFGRII and HFGRIII.

Additional file 5: Table S4. Filtered variants from metagenomic sequence analysis of HFGRII and HFGRIII.

Additional file 6: Figure S2. Copy number variation frequency spectrum at the CPRS locus of the 55th generation of HFGRIII (2-200x) cloned parasites. Of the parasites segregating at the 55th generation of HFGRIII, 71\% have three WT copies of CPRS. We tested 14 parasites cloned by dilution from the HFGIII 55th generation for CPRS amplification. Copy number variation was determined by $\triangle \triangle C t \mathrm{gPCR}$ using the $3^{\prime}{ }^{\prime} P R S$ assay. Seryl-tRNA synthetase served as the control gene and all copy number variation quantification was made in reference to a single CPRS copy Dd2 parasite.

Additional file 7: Table S5. Correlation between CPRS copy number and drug resistance. $R^{2}$ Pearson correlation coefficients of the relationship between CPRS copy number and the EC50 values of 11 antimalarial compounds. The parasites used for this analysis were HFGRIII 2-200x clone $\mathrm{H} 3$ (one copy), H6 (two copies), and A8 (three copies). $\mathrm{R}^{2}$ values range from 0 to 1 , representing little to strong correlation, respectively. *Two-tailed $t$-test $P$-value $<0.05$.

Additional file 8: Figure S5. Induction of halofuginone resistance in HB3 confirms proline-dependent induction of halofuginone resistance is not strain specific. (a) After eight generations of exposure to $4 \times \mathrm{EC} 50$ of halofuginone (2.8 nM), HB3-induced parasites recrudesced and returned 20- to 30-fold resistant to halofuginone treatment. $(b, c)$ Of the 19 proteogenic amino acids assayed, only proline concentrations were increased in HB3-induced parasites in saponin-released parasites (b) and purified iRBCs (c). EC50s were determined by SYBR Green growth assay. Fold increase in amino acid concentration upon induction or selection is expressed as a ratio of induced/selected line measurement over parental $\mathrm{Dd} 2$ line measurement. Amino acid levels of $P$. falciparum parasites were quantified from the normalized integrated peak intensity from LC-MS analysis. Error bars denote standard deviation.

Additional file 9: Figure S3. Induced Dd2 parasites show no evidence of genetic modifications at the CPRS locus. (a) Sanger sequencing across the CPRS allele revealed that induced and untreated Dd2 strains have WT CPRS alleles. The codon where the HFGRI (T14445A) and HFGRII (C1444T) mutations occurred is highlighted in yellow. (b) Expression of CPRS in synchronous early schizonts is invariant between untreated and induced Dd2 parasite lines.

Additional file 10: Table S6. SNPs called between whole-genome sequencing of Dd2 untreated and Dd2 Induced 1 and Dd2 Induced 2 lines.

Additional file 11: Figure S4. Halofuginone short-term induced long-term selection lines specifically upregulate intracellular proline concentration as measured in magnetically purified iRBCs. (a,b) Of the 19 proteogenic amino acids assayed, only proline concentrations were increased in Dd2 induced (a) and Dd2 long term selected (b) HFGRII and HFGRIII parasites within iRBCs. IRBC proline concentrations increased 4- to 6-fold compared with 20- to 30-fold in the corresponding saponin-released parasite samples. Fold-increase in amino acid concentration upon induction or selection is expressed as a ratio of induced/selected line measurement over parental Dd2 line measurement. Amino acid levels of RBCs infected with P. falciparum parasites were quantified from the normalized integrated peak intensity from LC-MS analysis. Error bars denote standard deviation.

Additional file 12: Figure S6. Early dynamics of proline and halofuginone resistance in Dd2-induced parasites. After eight generations of exposure to $4 \times E C 50$ of halofuginone $(2.8 \mathrm{nM})$, Dd2-induced parasites recrudesced. (a) As early as five generations post-recrudescence, Dd2-induced parasites had elevated intracellular proline levels that remained constantly elevated for an additional 55 generations. Proline levels of saponin-released $P$. falciparum parasites were quantified from the normalized integrated peak intensity from LC-MS analysis. (b) Dd2 parasites five generations post-recrudescence had also reached their maximum halofuginone resistance (EC50s 25 to $30 \mathrm{nM}$ ). Fifty-five generations later, the halofuginone dose-response in Dd2-induced parasites was unchanged. Error bars in (b) denote $95 \%$ confidence interval.

Additional file 13: Figure S7. Dose-dependent inhibition of $P$. falciparum growth is dependent on the concentration of free proline. SYBR Green dose response assays were performed with untreated (WT) Dd2 parasites that were put in media with four different proline concentrations at the start of the 72-h assay. The proline concentration of different in vitro culture medias is expressed relative to unmodified RPMI media. Error bars reflect standard deviations of triplicate experiments.

Additional file 14: Figure S8. Correlation of full metabolite profile with proline concentrations in halofuginone short-term induced and long-term selected parasites. Of the 115 polar metabolites measured, only pipercolic acid and cis/trans-hydroxyproline concentrations are highly correlated with cytosolic proline concentrations in short-term induced Dd2 parasites, short-term induced HB3 parasites, and long-term selected Dd2 lines HFGRI, II, and III. This heat map represents the correlation coefficients (r-values) between cytosolic concentrations of metabolites with that of proline in three collections of wild-type and modified parasites: short-term induced Dd2 parasites, short-term induced HB3 parasites, and long-term selected Dd2 HFGR lines. High correlation $(r=1)$ is represented by red while low correlation $(r=-1)$ is represented by blue.

Additional file 15: Figure S9. Expression analysis of Dd2-induced parasites. (a) qPCR-based expression analysis of the clag family of transport-involved genes demonstrates that their differential expression is not involved in Dd2 induced halofuginone resistance. Relative expression was calculated with the ddCt method using SerRS as control and in reference to a stage-matched untreated Dd2 parasite. FBP-Aldolase and ProRS (5') are provided as additional controls. Results represent eight biological replicate measurements (each data point) each determined by technical quadruplicate. (b) Spearman correlation was used to directly identify the stage of the three Dd2 RNA-Seq libraries assembled. (c) Log-transformed fold change of replicate Dd2 inductions (Dd2-Induced 1 and Dd2-Induced 2) (y-axis) was graphed with the logtransformed fold change from a 3D7 RNA-Seq control time course (x-axis) 
between corresponding stages (40 vs 36 hours post-infection ). By identifying outliers from this linear fitting, (d) we determined genes that are differential expressed between $\mathrm{Dd} 2$ induced and untreated parasites independent of stage-specific expression.

Additional file 16: Table S7. Quantitative PCR control primers for $\triangle \triangle C t$ analysis.

Additional file 17: Table S8. Quantitative PCR primers for the $C P R S$ target locus analysis.

Additional file 18: Table S9. Quantitative PCR primers for the clag gene family expression analysis.

\section{Abbreviations}

cPRS: cytoplasmic prolyl-tRNA synthetase; GATK: Genome Analysis Toolkit; HFGRII/III: long-term halofuginone drug resistance selection II/II; hpi: hours post-infection; iRBC: infected red blood cell; LC-MS: liquid crystallography-mass spectrometry; PCR: polymerase chain reaction; qPCR: quantitative PCR; SNP: single-nucleotide polymorphism; WT: wild type.

\section{Competing interests}

The authors declare that they have no competing interests.

\section{Authors' contributions}

$J D H, U R, R M$, and DFW designed the experiments. JDH performed the experiments. JDH, DPR, JS, AAD, EM, KMB and CBC analyzed the data. JDH and DFW prepared this manuscript. All authors read and approved the final manuscript.

\section{Acknowledgements}

We would like to thank S Bopp for helpful discussion and comments on the manuscript. We would like to acknowledge JF Cortese for his contributions to the halofuginone project.

We gratefully acknowledge financial support for this work by the Gates Foundation (DFW and RM), Exxon Mobil Foundation, NSF Graduate Research Fellowship (DPR), and the National Institute of Health (DFW - Al084031, JDH - T32 GM007306-39, DPR and MMD - GM104239).

\section{Author details}

${ }^{1}$ Department of Immunology and Infectious Disease, Harvard School of Public Health, Boston, MA 02115, USA. ${ }^{2}$ Broad Institute of MIT and Harvard, Cambridge, MA 02142, USA. ${ }^{3}$ Biological and Biomedical Sciences, Boston, MA 02115, USA. ${ }^{4}$ Harvard/MIT Division of Health Sciences and Technology, Boston, MA 02115, USA. ${ }^{5}$ Harvard/MIT MD-PhD Program, Harvard Medical School, Boston, MA 02115, USA. ${ }^{\circ}$ Department of Organismic and Evolutionary Biology, Harvard University, Cambridge, MA 02138, USA. ${ }^{7}$ FAS Center for Systems Biology, Harvard University, Cambridge, MA 02138, USA. ${ }^{8}$ Department of Cell and Molecular Biology, Microbiology, Uppsala University, Uppsala 75105, Sweden. ${ }^{9}$ Massachusetts General Hospital, Center for Systems Biology, Boston, MA 02114, USA

Received: 1 October 2014 Accepted: 28 October 2014

Published online: 14 November 2014

\section{References}

1. Murray CJL, Rosenfeld LC, Lim SS, Andrews KG, Foreman KJ, Haring D, Fullman N, Naghavi M, Lozano R, Lopez AD: Global malaria mortality between 1980 and 2010: a systematic analysis. Lancet 2012, 379:413-431.

2. Noedl H, Se Y, Schaecher K, Smith BL, Socheat D, Fukuda MM, Artemisinin Resistance in Cambodia 1 (ARC1) Study Consortium: Evidence of artemisinin-resistant malaria in western Cambodia. N Engl J Med 2008, 359:2619-2620.

3. Ariey F, Witkowski B, Amaratunga C, Beghain J, Langlois A-C, Khim N, Kim S, Duru V, Bouchier C, Ma L, Lim P, Leang R, Duong S, Sreng S, Suon S, Chuor CM, Bout DM, Ménard S, Rogers WO, Genton B, Fandeur T, Miotto O, Ringwald P, Le Bras J, Berry A, Barale J-C, Fairhurst RM, Benoit-Vical F, Mercereau-Puijalon O, Ménard D: A molecular marker of artemisininresistant Plasmodium falciparum malaria. Nature 2014, 505:50-55.

4. Dong C, Urgaonkar S, Cortese J, Gamo F: Identification and validation of tetracyclic benzothiazepines as Plasmodium falciparum Cytochrome bc1 inhibitors. Chem Biol 2011, 18:1602-1610.
5. Sidhu A, Sun $Q$, Nkrumah $L$, Dunne M: In vitro efficacy, resistance selection, and structural modeling studies implicate the malarial parasite apicoplast as the target of azithromycin. J Biol 2007, 282:2494-2504

6. Rottmann M, McNamara C, Yeung BKS, Lee MCS, Zou B, Russell B, Seitz P, Plouffe DM, Dharia NV, Tan J, Cohen SB, Spencer KR, González-Páez GE, Lakshminarayana SB, Goh A, Suwanarusk R, Jegla T, Schmitt EK, Beck HP, Brun R, Nosten F, Renia L, Dartois V, Keller TH, Fidock DA, Winzeler EA, Diagana TT: Spiroindolones, a potent compound class for the treatment of malaria. Science 2010, 329:1175-1180.

7. Hoepfner D, McNamara CW, Lim CS, Studer C, Riedl R, Aust T, McCormack SL, Plouffe DM, Meister S, Schuierer S, Plikat U, Hartmann N, Staedtler F, Cotesta S, Schmitt EK, Petersen F, Supek F, Glynne RJ, Tallarico JA, Porter JA, Fishman MC, Bodenreider C, Diagana TT, Movva NR, Winzeler EA: Selective and specific inhibition of the plasmodium falciparum lysyl-tRNA synthetase by the fungal secondary metabolite cladosporin. Cell Host Microbe 2012, 11:654-663.

8. Harbut MB, Patel BA, Yeung BKS, McNamara CW, Bright AT, Ballard J, Supek F, Golde TE, Winzeler EA, Diagana TT, Greenbaum DC: Targeting the ERAD pathway via inhibition of signal peptide peptidase for antiparasitic therapeutic design. Proc Natl Acad Sci U S A 2012, 109:21486-21491.

9. Lukens AK, Ross LS, Heidebrecht R, Javier Gamo F, Lafuente-Monasterio MJ, Booker ML, Hartl DL, Wiegand RC, Wirth DF: Harnessing evolutionary fitness in Plasmodium falciparum for drug discovery and suppressing resistance. Proc Natl Acad Sci U S A 2013, 11:799-804.

10. Flannery EL, Chatterjee AK, Winzeler EA: Antimalarial drug discoveryapproaches and progress towards new medicines. Nat Rev Microbiol 2013, 11:849-862.

11. Gardner MJ, Hall N, Fung E, White O, Berriman M, Hyman RW, Carlton JM, Pain A, Nelson KE, Bowman S, Paulsen IT, James K, Eisen JA, Rutherford K, Salzberg SL, Craig A, Kyes S, Chan M-S, Nene V, Shallom SJ, Suh B, Peterson J, Angiuoli S, Pertea M, Allen J, Selengut J, Haft D, Mather MW, Vaidya AB, Martin DMA, et al: Genome sequence of the human malaria parasite Plasmodium falciparum. Nature 2002, 419:498-511.

12. Weinreich DM, Delaney NF, Depristo MA, Hartl DL: Darwinian evolution can follow only very few mutational paths to fitter proteins. Science 2006, 312:111-114.

13. Woods RJ, Barrick JE, Cooper TF, Shrestha U, Kauth MR, Lenski RE: Secondorder selection for evolvability in a large Escherichia coli population. Science 2011, 331:1433-1436.

14. Plucain J, Hindré T, Le Gac M, Tenaillon O, Cruveiller S, Médigue C, Leiby N, Harcombe WR, Marx CJ, Lenski RE, Schneider D: Epistasis and allele specificity in the emergence of a stable polymorphism in Escherichia coli. Science 2014, 343:1366-1369.

15. Blount ZD, Borland CZ, Lenski RE: Historical contingency and the evolution of a key innovation in an experimental population of Escherichia coli. Proc Natl Acad Sci U S A 2008, 105:7899-7906.

16. Bloom JD, Gong LI, Baltimore D: Permissive secondary mutations enable the evolution of influenza oseltamivir resistance. Science 2010, 328:1272-1275.

17. Rokyta DR, Joyce P, Caudle SB, Miller C, Beisel CJ, Wichman HA: Epistasis between beneficial mutations and the phenotype-to-fitness map for a ssDNA virus. PLoS Genet 2011, 7:e1002075.

18. Burch $\mathrm{CL}$, Chao L: Evolvability of an RNA virus is determined by its mutational neighbourhood. Nature 2000, 406:625-628.

19. Barrick JE, Yu DS, Yoon SH, Jeong H, Oh TK, Schneider D, Lenski RE, Kim JF: Genome evolution and adaptation in a long-term experiment with Escherichia coli. Nature 2009, 461:1243-1247.

20. Lang Gl, Rice DP, Hickman MJ, Sodergren E, Weinstock GM, Botstein D, Desai MM: Pervasive genetic hitchhiking and clonal interference in forty evolving yeast populations. Nature 2013, 500:571-574.

21. Kryazhimskiy S, Rice DP, Jerison E, Desai MM: Global Epistasis Makes Adaptation Predictable Despite Sequence-Level Stochasticity; 2014.

22. Pham JS, Dawson KL, Jackson KE, Lim EE, Pasaje CFA, Turner KEC, Ralph SA Aminoacyl-tRNA synthetases as drug targets in eukaryotic parasites. Int $J$ Parasitol 2013, 4:1-13.

23. Istvan ES, Dharia NV, Bopp SE, Gluzman I, Winzeler EA, Goldberg DE: Validation of isoleucine utilization targets in Plasmodium falciparum. Proc Natl Acad Sci U S A 2011, 108:1627-1632.

24. Habibi D, Ogloff N, Jalili RB, Yost A, Weng AP, Ghahary A, Ong CJ: Borrelidin, a small molecule nitrile-containing macrolide inhibitor of threonyl-tRNA synthetase, is a potent inducer of apoptosis in acute lymphoblastic leukemia. Invest New Drugs 2011, 30:1361-1370. 
25. Bopp SER, Manary MJ, Bright AT, Johnston GL, Dharia NV, Luna FL, McCormack S, Plouffe D, McNamara CW, Walker JR, Fidock DA, Denchi EL, Winzeler EA: Mitotic evolution of Plasmodium falciparum shows a stable core genome but recombination in antigen families. PLoS Genet 2013, 9:e1003293.

26. Zhou H, Sun L, Yang X-L, Schimmel P: ATP-directed capture of bioactive herbal-based medicine on human tRNA synthetase. Nature 2012, 494:121-124.

27. Keller TL, Zocco D, Sundrud MS, Hendrick M, Edenius M, Yum J, Kim Y-J, Lee H-K, Cortese JF, Wirth DF, Dignam JD, Rao A, Yeo C-Y, Mazitschek R, Whitman M: Halofuginone and other febrifugine derivatives inhibit prolyl-tRNA synthetase. Nat Chem Biol 2012, 8:311-317.

28. He M: Pipecolic acid in microbes: biosynthetic routes and enzymes. J Ind Microbiol Biotechnol 2006, 33:401-407.

29. Ginsburg H, Kutner S, Krugliak M, loav Cabantchik Z: Characterization of permeation pathways appearing in the host membrane of plasmodium falciparum infected red blood cells. Mol Biochem Parasitol 1985, 14:313-322.

30. Kutner S, Breuer WW, Ginsburg H, Aley SB, Cabantchik Zl: Characterization of permeation pathways in the plasma membrane of human erythrocytes infected with early stages of Plasmodium falciparum: association with parasite development. J Cell Physiol 1985, 125:521-527.

31. McCormick GJ: Amino acid transport and incorporation in red blood cells of normal and Plasmodium knowlesi-infected rhesus monkeys. Exp Parasitol 1970, 27:143-149.

32. Desai SA: Why do malaria parasites increase host erythrocyte permeability? Trends Parasitol 2014, 30:151-159.

33. Mira-Martínez S, Rovira-Graells N, Crowley VM, Altenhofen LM, Llinás M, Cortés A: Epigenetic switches in clag3 genes mediate blasticidin S resistance in malaria parasites. Cell Microbiol 2013, 15:1913-1923.

34. Sharma P, Wollenberg K, Sellers M, Zainabadi K, Galinsky K, Moss E, Nguitragool W, Neafsey D, Desai SA: An epigenetic antimalarial resistance mechanism involving parasite genes linked to nutrient uptake. J Biol Chem 2013, 288:19429-19440.

35. Ganesan K, Ponmee N, Jiang L, Fowble JW, White J, Kamchonwongpaisan S, Yuthavong Y, Wilairat P, Rathod PK: A genetically hard-wired metabolic transcriptome in Plasmodium falciparum fails to mount protective responses to lethal antifolates. PLOS Pathog 2008, 4:e1000214.

36. Shafqat S, Velaz-Faircloth M, Henzi VA, Whitney KD, Yang-Feng TL, Seldin MF, Fremeau RT: Human brain-specific L-proline transporter: molecular cloning, functional expression, and chromosomal localization of the gene in human and mouse genomes. Mol Pharmacol 1995, 48:219-229.

37. Kowalczuk S, Bröer A, Munzinger M, Tietze N, Klingel K, Bröer S: Molecular cloning of the mouse IMINO system: an $\mathrm{Na}+-$ and $\mathrm{Cl}$-dependent proline transporter. Biochem J 2005, 386:417-422.

38. Caspi R, Altman T, Dale JM, Dreher K, Fulcher CA, Gilham F, Kaipa P, Karthikeyan AS, Kothari A, Krummenacker M, Latendresse M, Mueller LA, Paley S, Popescu L, Pujar A, Shearer AG, Zhang P, Karp PD: The MetaCyc database of metabolic pathways and enzymes and the BioCyc collection of pathway/genome databases. Nucleic Acids Res 2010, 38:D473-479.

39. Cao B, Li M, Zha W, Zhao Q, Gu R, Liu L, Shi J, Zhou J, Zhou F, Wu X, Wu Z, Wang G, Aa J: Metabolomic approach to evaluating adriamycin pharmacodynamics and resistance in breast cancer cells. Metabolomics 2013, 9:960-973.

40. Martinez-Outschoorn UE, Lin Z, Ko Y-H, Goldberg AF, Flomenberg N, Wang C, Pavlides S, Pestell RG, Howell A, Sotgia F, Lisanti MP: Understanding the metabolic basis of drug resistance: therapeutic induction of the Warburg effect kills cancer cells. Cell Cycle 2011, 10:2521-2528.

41. Ryu CS, Kwak HC, Lee KS, Kang KW, Oh SJ, Lee KH, Kim HM, Ma JY, Kim SK: Sulfur amino acid metabolism in doxorubicin-resistant breast cancer cells. Toxicol Appl Pharmacol 2011, 255:94-102.

42. Ryu CS, Kwak HC, Lee J-Y, Oh SJ, Phuong NTT, Kang KW, Kim SK: Elevation of cysteine consumption in tamoxifen-resistant MCF-7 cells. Biochem Pharmacol 2013, 85:197-206.

43. Fennell C, Babbitt S, Russo I, Wilkes J, Ranford-Cartwright L, Goldberg DE, Doerig C: PfelK1, a eukaryotic initiation factor 2alpha kinase of the human malaria parasite Plasmodium falciparum, regulates stress-response to amino-acid starvation. Malar J 2009, 8:99.

44. Babbitt SE, Altenhofen L, Cobbold SA, Istvan ES, Fennell C, Doerig C, Llinás $\mathrm{M}$, Goldberg DE: Plasmodium falciparum responds to amino acid starvation by entering into a hibernatory state. Proc Natl Acad Sci U S A 2012, 109:E3278-E3287.

45. Björkman J, Nagaev I, Berg OG, Hughes D, Andersson DI: Effects of environment on compensatory mutations to ameliorate costs of antibiotic resistance. Science 2000, 287:1479-1482.

46. Levin BR, Perrot V, Walker N: Compensatory mutations, antibiotic resistance and the population genetics of adaptive evolution in bacteria. Genetics 2000, 154:985-997.

47. Weisberg E, Manley PW, Cowan-Jacob SW, Hochhaus A, Griffin JD: Second generation inhibitors of BCR-ABL for the treatment of imatinib-resistant chronic myeloid leukaemia. Nat Rev Cancer 2007, 7:345-356.

48. Johnson JD, Dennull RA, Gerena L, Lopez-Sanchez M, Roncal NE, Waters NC: Assessment and continued validation of the malaria SYBR green I-based fluorescence assay for use in malaria drug screening. Antimicrob Agents Chemother 2007, 51:1926-1933.

49. Desjardins RE, Canfield CJ, Haynes JD, Chulay JD: Quantitative assessment of antimalarial activity in vitro by a semiautomated microdilution technique. Antimicrob Agents Chemother 1979, 16:710-718.

50. Trager W, Jensen J: Human malaria parasites in continuous culture. Science 1976, 193:673-675.

51. Bei AK, Desimone TM, Badiane AS, Ahouidi AD, Dieye T, Ndiaye D, Sarr O, Ndir O, Mboup S, Duraisingh MT: A flow cytometry-based assay for measuring invasion of red blood cells by Plasmodium falciparum. Am J Hematol 2010, 85:234-237.

52. Townsend MK, Clish CB, Kraft P, Wu C, Souza AL, Deik AA, Tworoger SS, Wolpin BM: Reproducibility of metabolomic profiles among men and women in 2 large cohort studies. Clin Chem 2013, 59:1657-1667.

doi:10.1186/s13059-014-0511-2

Cite this article as: Herman et al:: A genomic and evolutionary approach reveals non-genetic drug resistance in malaria. Genome Biology 2014 15:511.

\section{Submit your next manuscript to BioMed Central and take full advantage of:}

- Convenient online submission

- Thorough peer review

- No space constraints or color figure charges

- Immediate publication on acceptance

- Inclusion in PubMed, CAS, Scopus and Google Scholar

- Research which is freely available for redistribution

Submit your manuscript at www.biomedcentral.com/submit
C Biomed Central 